Quim. Nova, Vol. 34, No. 2, 250-255, 2011

\title{
DEVELOPMENT AND VALIDATION OF A STABILITY-INDICATING UPLC METHOD FOR ROSUVASTATIN AND ITS RELATED IMPURITIES IN PHARMACEUTICAL DOSAGE FORMS
}

\author{
Gosula Venkat Ram Reddy, Bobba Venkateswara Reddy, Syed Wasimul Haque, Haum Dutt Gautam and Poonam Kumar \\ Jubilant Organosys Limited D-12, Sector 59 Noida, Uttar Pradesh, India \\ Avvaru Praveen Kumar* and Jung Hag Park \\ Department of Chemistry, Yeungnam University, 214-1 Dae-dong, Gyeongsan 712-749, Korea
}

Recebido em 10/3/10; aceito em 19/8/10; publicado na web em 30/11/10

\begin{abstract}
The present work describes a novel stability-indicating reversed-phase ultra performance liquid chromatography method for the separation and quantification of rosuvastatin (RSV) and its related impurities in the pharmaceutical dosage forms under forced degradation conditions. An unknown degradation impurity detected in the acid degradation was identified by using quadrupole time-of-flight mass spectrometry. The chromatographic separation was carried out on C-18 column ( $100 \times 2.1 \mathrm{~mm}, 1.7 \mu \mathrm{m})$ using isocratic elution with methanol and $0.1 \%$ trifluoroacetic acid (50:50). The total run time was $12 \mathrm{~min}$ within which RSV as well as all related impurities and degradation products were separated. The developed method was validated for RSV and related impurities in pharmaceutical dosage forms.
\end{abstract}

Keywords: UPLC-QToF-MS; rosuvastatin tablets; degradation study.

\section{INTRODUCTION}

Rosuvastatin (RSV) (Figure 1) is a synthetic lipid-lowering agent, chemically known as $(3 R, 5 S, 6 E)$-7-[4-(4-fluorophenyl)-6(1-methylethyl)-2-[methyl(methylsulfonyl)amino]-5-pyrimidinyl]3,5-dihydroxy-6-heptenoicacid, calcium salt (2:1). It is used for the treatment of hyperlipidemia and is an inhibitor of 3-hydroxy-3-methylglutaryl coenzyme (HMG-CoA) reductase. This enzyme catalyzes the conversion of HMG-CoA to mevalonate, an early and rate-limiting step in cholesterol biosynthesis. ${ }^{1} \mathrm{RSV}$ calcium is a salt with $\mathrm{pK}_{\mathrm{a}}$ of 4.6 and very slightly soluble in aqueous solutions of $\mathrm{pH} 4.0$ and below. Compared with other HMG-CoA inhibitors, RSV Ca does not appear to be metabolized significantly by cytochrome $\mathrm{P} 4503 \mathrm{~A} 4{ }^{2}$ and therefore, may not possess the same potential for drug interaction as seen for some other statins, e.g. lovastatin and simvastatin. ${ }^{3,4}$ It exhibits a high degree of specificity for uptake into the liver and is a potent in vitro and in vivo competitive inhibitor of HMG-CoA reductase. Anti-isomer of $\mathrm{RSV}^{5}$ and lactone impurities are the major process-related impurities of RSV and their chemical structures are shown in Figure 1.

Ultra performance liquid chromatography (UPLC) has been considered as a novel development in liquid chromatography. It is specially designed to withstand higher system pressures during chromatographic analysis so that it enables significant decrease in separation time and solvent consumption. The UPLC columns packed with 1.7 $\mu \mathrm{m}$ sized particles provides not only increased efficiency but also the ability to work at increased linear velocity without loss of efficiency, providing both resolution and speed. Using advantages of UPLC, a number of applications in different fields including pharmacy, ${ }^{6}$ clinical analysis, pesticide analysis ${ }^{7}$ and tetracyclines in human urine ${ }^{8}$ have been reported. For structural elucidation accurate mass data gives the reliable information which can confirm the molecular formula of a compound. ${ }^{9}$ QToF-MS provides exact mass measurements for both precursor and fragment ions to yield high confidence in structural elucidation. ${ }^{10}$ The coupling of UPLC with QToF-MS/MS extends high

*e-mail: drkumar.kr@gmail.com chromatographic resolution with exact mass measurement for both MS and MS/MS, and then allows significant advantages of speed, selectivity, sensitivity and accuracy for rapid screening of known as well as unknown compounds.

Different analytical methods were reported for determination of RSV by capillary zone electrophoresis, ${ }^{11} \mathrm{UV}$ spectrophotometry, ${ }^{12,13}$ automated solid phase extraction using mass spectrometry ${ }^{14}$ and ion pair liquid-liquid extraction employing liquid chromatography with electrospray ionization tandem mass spectrometry. ${ }^{15}$ Mehta et al. reported a stability-indicating assay method for determination of RSV in presence of its degradation products using high performance liquid chromatography (HPLC). ${ }^{16}$ In their assay method, run time was around 35 min to elute all degradation impurities and applicable for only RSV estimation but not for its related substances. In the literature, there were no UPLC methods available to estimate related substances of RSV and its assay during stability-indicating analysis in pharmaceutical dosage forms.

The aim of the present work was the development of a stabilityindicating UPLC method for the determination of RSV and its related impurities in the pharmaceutical dosage forms under forced degradation conditions. The developed method was validated according to International Conference on Harmonization (ICH) guidelines ${ }^{17}$ to show the stability indicating capability of the method.

\section{EXPERIMENTAL}

\section{Reagents and chemicals}

Reference standards of RSV calcium, anti-isomer and lactone impurities were gifted by Jubilant Organosys limited (Noida, India) with declared purities of 99.1 and 92.7 and $85.9 \%$, respectively. Methanol (MeOH) of HPLC grade was received from J. T. Baker (NJ, USA). Potassium dihydrogen phosphate $\left(\mathrm{KH}_{2} \mathrm{PO}_{4}\right)$ and trifluoroacetic acid (TFA) were obtained from Qualigens fine chemicals (Mumbai, India). All solutions were filtered through $0.2 \mu$ m nylon filters manufactured by Millipore Pvt. Ltd (Bangalore, India). Tablet 
(a)

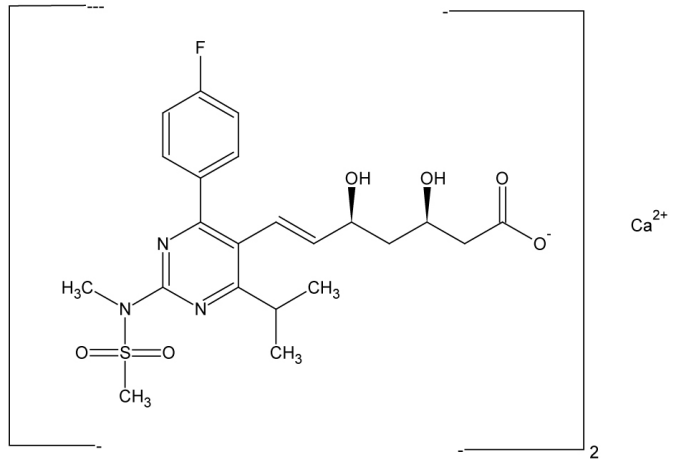

(b)

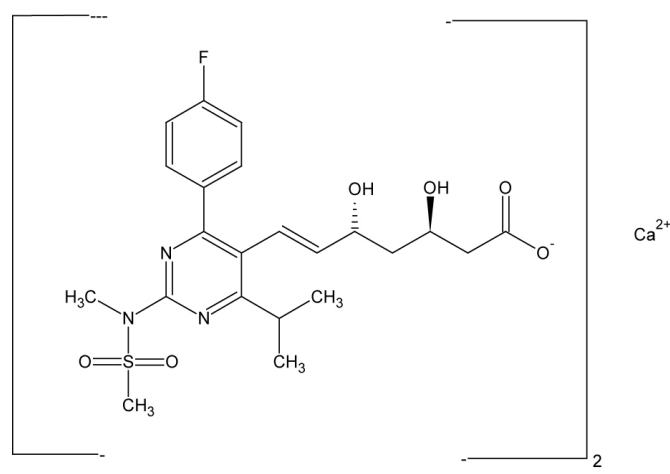

(c)<smiles>CC(C)c1nc(N(C)S(C)(=O)=O)nc(-c2ccc(F)cc2)c1/C=C/C1CC(O)CC(=O)O1</smiles>

Figure 1. Structures of RSV (a), its anti-isomer (b) and lactone (c) impurities

formulations containing 5 and $40 \mathrm{mg}$ of RSV were obtained from Jubilant Organosys limited (Noida, India).

\section{Chemical names of RSV and its related impurities}

RSV: (3R,5S,6E)-7-[4-(4-fluorophenyl)-6-(1-methylethyl)-2[methyl(methylsulfonyl)amino]-5-pyrimidinyl]-3,5-dihydroxy-6heptenoic acid.

Anti-isomer impurity: (3R,5R)-7-[4-(4-fluorophenyl)-6isopropyl-2-[methyl(methylsulfonyl) amino]pyrimidin-5-yl]-3,5dihydroxy-6(E)-heptenoic acid.

Lactone impurity: $N$-\{4-(4-fluoro-phenyl)-5-[2-(4-hydroxy-6oxo-tetrahydro-pyran-2-yl)-vinyl]-6-isopropyl-pyrimidin-2-yl $\}-N$ methyl-methanesulfonamide.

\section{Equipment}

Analysis was performed on Waters Acquity UPLC ${ }^{\mathrm{TM}}$ system (Milford, USA) consists of a binary solvent manager, a sample manager and a photodiode array (PDA) detector. Cintex digital water bath was used for acid and base hydrolysis studies. Photostability studies were carried out in a Sanyo photostability chamber (Leicestershire, UK). Thermal stability studies were performed in a dry air oven from Cintex (Mumbai, India). Intermediate precision study was performed on different Waters Acquity UPLC ${ }^{\mathrm{TM}}$ system (Milford, USA) consists of a binary solvent manager, a sample manager and a tunable ultraviolet (TUV) detector.

\section{Chromatographic conditions}

The mobile phase used was a mixture of $\mathrm{MeOH}$ and $0.1 \%$ TFA in the ratio of 50:50 employing isocratic elution. The detector was set at a sampling rate of 20 points $\mathrm{s}^{-1}$ and filter time constant of 0.2 s. System control, data collection and data processing were accomplished using Waters Empower chromatography data software. The analytical column used was $100 \times 2.1 \mathrm{~mm}, 1.7 \mu \mathrm{m}$ Waters acquity UPLC BEH C-18 column (Milford, USA). The optimized conditions were as follows: injection volume: $2.0 \mu \mathrm{L}$, flow rate: $0.3 \mathrm{~mL} \mathrm{~min}^{-1}$ at a column temperature of $40^{\circ} \mathrm{C}$, sample cooler temperature: $10^{\circ} \mathrm{C}$ and detection wavelength: $240 \mathrm{~nm}$. Under these conditions the system back pressure was about 7200 psi.

\section{UPLC-MS conditions}

To identify the unknown degradant formed in the acid degradation sample UPLC-MS/MS analysis was performed using Waters Acquity UPLC $^{\mathrm{TM}}$ system connected to QToF mass spectrometer from Waters Corporation (Milford, USA). Mobile phase composed of $0.1 \%$ TFA and $\mathrm{MeOH}$ in the ratio of 50:50 with a flow rate of $0.3 \mathrm{~mL} \mathrm{~min}{ }^{-1}$. The analysis was performed using electrospray ionization in positive mode and operating parameters as follows: sample cone voltage: $30 \mathrm{~V}$, capillary voltage: $3500 \mathrm{~V}$ and extraction cone voltage: $1.0 \mathrm{~V}$. Source temperature set at $100{ }^{\circ} \mathrm{C}$ and desolvation temperature at $250{ }^{\circ} \mathrm{C}$.

\section{Preparation of solutions}

\section{Standard stock solutions}

The individual standard stock solutions of RSV, its anti-isomer and lactone impurities were prepared by dissolving corresponding standard compounds in diluent (Diluent was 50:50 of $\mathrm{MeOH}$ and water) to obtain a concentration of 500,50 and $50 \mu \mathrm{g} \mathrm{mL}^{-1}$, respectively. From the standard stock solution of RSV, $5 \mathrm{~mL}$ was diluted to $50 \mathrm{~mL}$ with diluent and used as the assay standard. Five $\mathrm{mL}$ of this assay standard solution was further diluted to $50 \mathrm{~mL}$ with diluent and used as the standard to quantify the related impurities.

\section{System suitability solution}

System suitability parameters were measured in order to verify the system performance. The system suitability solution was prepared to obtain 500,5 and $5 \mu \mathrm{g} \mathrm{mL}^{-1}$ concentrations of RSV, anti-isomer and lactone, respectively. Six replicate injections of the system suitability solution and diluted RSV standard solution were performed to measure the important characteristics including peak resolution, tailing factor and theoretical plates.

\section{Sample preparation}

Taken twenty tablets of both strengths ( 5 and $40 \mathrm{mg}$ of RSV) and made it as fine powder. Transferred tablet powder equivalent to $50 \mathrm{mg}$ of RSV into a $100 \mathrm{~mL}$ volumetric flask, added $70 \mathrm{~mL}$ of the diluent and sonicate for $30 \mathrm{~min}$. Keep the solution aside to attain room temperature, then make up to the volume with diluent and filtered through 
$0.2 \mu \mathrm{m}$ nylon filter. This solution was employed as sample solution for RSV related impurities. Five $\mathrm{mL}$ of this solution was diluted to $50 \mathrm{~mL}$ with diluent and filtered, used as the assay sample solution.

\section{Specificity}

Forced degradation studies were performed to demonstrate selectivity and stability-indicating capability of the proposed method. The powdered tablet sample and placebo were exposed to acid $(1 \mathrm{~N}$ $\mathrm{HCl}, 3 \mathrm{~h})$, base $\left(1 \mathrm{~N} \mathrm{NaOH}, 70{ }^{\circ} \mathrm{C}, 2 \mathrm{~h}\right)$, strong oxidation $\left(5 \% \mathrm{H}_{2} \mathrm{O}_{2}\right.$, $70{ }^{\circ} \mathrm{C}, 2 \mathrm{~h}$ ), thermal $\left(105{ }^{\circ} \mathrm{C}, 7\right.$ days $)$, humidity $\left(90 \% \mathrm{RH}, 25{ }^{\circ} \mathrm{C}, 7\right.$ days) and photolytic (1.2 million lux h, $200 \mathrm{wh} / \mathrm{m}^{2}, 7$ days) degradation conditions. Samples were withdrawn at appropriate times and subjected to UPLC analysis after suitable dilution $\left(500 \mu \mathrm{g} \mathrm{mL}^{-1}\right)$ to evaluate the ability of the proposed method to separate RSV from its degradation products and placebo. Photodiode array detector was employed to check and ensure the homogeneity and purity of RSV peak in all the stressed sample solutions.

\section{Linearity}

Linearity test solutions of related impurities were prepared from the impurities stock solution at five different concentration levels in triplicate from LOQ to $150 \%$ of the specification (LOQ, 50, 75, 100 and $150 \%$ ) to the impurities level. The calibration curves were constructed by plotting peak areas of impurity versus its corresponding concentrations. The slope, Y-intercept and correlation coefficient of the calibration curve were calculated for all the impurities.

Solutions for assay linearity were prepared from RSV stock solution at 5 different concentration levels ranging from 50-150\% of assay sample concentration.

\section{Limit of detection (LOD) and limit of quantification (LOQ)}

The LOD and LOQ of RSV and its impurities were determined by using the signal to noise approach as defined in $\mathrm{ICH}$ guidelines. ${ }^{17}$

\section{Precision}

\section{System precision}

System precision was evaluated by injecting 6 replicate injections of the system suitability solution and calculated the \% RSD for each impurity.

\section{Method precision}

Method precision was evaluated by injecting the 6 different preparations of the system suitability solution and calculated the $\%$ RSD for each impurity.

Method precision for assay was performed by injecting six independent assay preparations of RSV sample against qualified working standard and calculated the \% RSD.

\section{Accuracy}

Recovery experiments were carried out to confirm the accuracy of the proposed method. The accuracy of the assay method was evaluated at 4 different concentration levels of LOQ, 50, 100 and $150 \%$ to the specification limits in triplicate. The samples were analyzed by the proposed method and the percentage recoveries for each and every impurity at each level and each replicate were calculated.

For assay, recovery experiments were carried out by preparing the solutions at 50,100 and $150 \%$ to the test concentration by taking the tablet powder equivalent to $25 \mathrm{mg}$ of RSV, $50 \mathrm{mg}$ of RSV and
$75 \mathrm{mg}$ of RSV in different $100 \mathrm{~mL}$ flasks. The percentage recoveries were calculated.

\section{Robustness}

The robustness as a measure of method capability to remain unaffected by small, but deliberate changes in chromatographic conditions was studied by testing influence of small changes in mobile phase composition $\left(2 \%\right.$ absolute change), column temperature $\left( \pm 5^{\circ} \mathrm{C}\right)$ and flow rate $\left( \pm 0.1 \mathrm{~mL} \mathrm{~min}^{-1}\right)$.

\section{Stability of sample preparation}

Stability of sample solution was established by storage of sample solution at ambient temperature $\left(25^{\circ} \mathrm{C}\right)$ for $24 \mathrm{~h}$.

\section{RESULT AND DISCUSSION}

\section{Method development and optimization}

During the literature survey of the RSV assay, there were no methods available for estimation of related impurities of RSV in its drug product except a stability-indicating assay method for the determination of RSV in presence of its degradation products using HPLC. ${ }^{16}$ The present work was aimed to develop a stability-indicating UPLC method for the determination of RSV and its related impurities in the tablet formulations. At the beginning, the method development process was carried by employing a sample solution contains 500 , 5 and $5 \mu \mathrm{g} \mathrm{mL}^{-1}$ of RSV, anti-isomer and lactone, respectively. The separation was performed on $50 \times 2.1 \mathrm{~mm}, 1.7 \mu \mathrm{m}$ UPLC BEH C-18 column by reversed-phase UPLC using a mobile phase of $0.01 \mathrm{M}$ $\mathrm{KH}_{2} \mathrm{PO}_{4}$ buffer ( $\left.\mathrm{pH} 3.0\right)$ and $\mathrm{MeOH}$ in the ratio of 40:60. It has been observed that the lactone impurity was well separated from the main peak (RSV) but anti-isomer was co-eluted with RSV.

Different parameters were investigated to separate the anti-isomer impurity from the RSV including different organic ratios, column lengths and additives in the buffer. The decreased organic ratio in mobile phase resulted in a poor separation of anti-isomer. The increased column length from 50 to $100 \mathrm{~mm}$ was found to be good for separation of anti-isomer from RSV but peak tailing was observed to RSV. In order to get better peak shape the potassium buffer was replaced with $0.1 \%$ TFA and carried the analysis. The anti-isomer and lactone impurities were clearly separated from the main peak with a resolution of 3.2 and 7.5, respectively, within a total run time of 7.5 min using isocratic elution. However, in specificity study of RSV from acid degradation, some non-polar degradants are detected. To elute these non-polar degradation impurities different isocratic and gradient runs were attempted. In one of the trials contains the mobile phase composition of buffer (0.1\% TFA): $\mathrm{MeOH}$ (50:50), all the known impurities and the unknown degradants were separated with good peak shapes and resolutions of not less than 1.5. A detection wavelength of $240 \mathrm{~nm}$ was selected because at this wavelength all degradation impurities show similar response. A typical chromatogram showing the separation of RSV and its known impurities was presented in Figure 2 and the chromatographic data were presented in Table 1.

\section{Forced degradation study results}

The degradation study revealed that RSV was very sensitive to acid hydrolysis compared to other degradation conditions. The RSV and its placebo were treated with different degradation conditions including acid, base, peroxide, photolytic, humidity and thermal degradations, the respective distinct chromatograms were shown 
Table 1. Chromatographic performance data

\begin{tabular}{lcccccc}
\hline Compound & $\mathrm{t}_{\mathrm{R}}(\mathrm{min})$ & $\mathrm{RRT}^{\mathrm{a}, \mathrm{b}}$ & Resolution $^{\mathrm{b}, \mathrm{c}}$ & Tailing factor $^{\mathrm{b}}$ & Theoretical plates $^{\mathrm{b}}$ & RRF $^{\mathrm{b}, \mathrm{d}}$ \\
\hline RSV & 5.41 & -- & -- & 1.16 & 13000 & - \\
Anti-isomer & 6.17 & 1.14 & 3.11 & 0.92 & 11000 & 0.94 \\
Lactone & 7.41 & 1.37 & 8.38 & 1.12 & 10500 & 0.91 \\
\hline
\end{tabular}

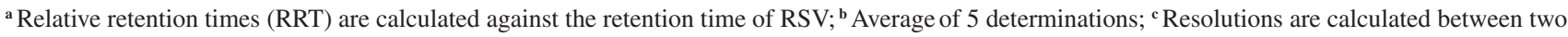
adjacent peaks; ${ }^{\mathrm{d}}$ Relative response factor was calculated using slope method.

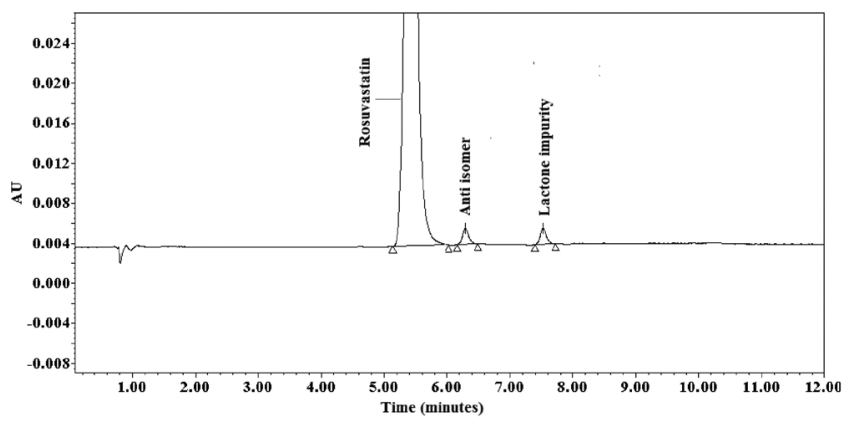

Figure 2. Separation of RSV and its related impurities

in Figure 3. The major degradation products of acid hydrolysis are lactone and an unknown impurity, detected at the retention times of 7.517 and $9.835 \mathrm{~min}$, respectively. The forced degradation results were shown in Table 2, indicate that RSV is considerably stable under neutral and basic conditions. The degradation of RSV in thermal, humidity and photolytic conditions was observed to be lower. Further, the spectra of the unknown impurities formed in the degradation of RSV were similar to that of the individual standards eluting at respective retention times. Also the spectra of the known impurities in the degraded tablet sample were similar to its respective impurity standard substance, indicating that there was no co-elution of unknown degradation peak at the retention time of the respective known and unknown impurities. Spectral purity of RSV and its impurities in the chromatogram of all the exposed samples are obtained from PDA and found to be spectrally pure. The max plot chromatogram of degradation sample was also checked to ensure that no degradation peak is missed due to use of wavelength of $240 \mathrm{~nm}$.

For assay, the forced degradation study was performed by injecting the degradation samples at the assay concentration level i.e. 50 $\mu \mathrm{g} \mathrm{mL} \mathrm{m}^{-1}$ and calculated the assay percentage against the reference standard. A summation of assay value and degradation impurity levels generally may be used to obtain mass balance for the test sample and to see how closely these add up to $100 \%$ of initial value with due consideration of the margin of analytical error. ${ }^{18}$ Mass balance was calculated for all the degradation samples by summing all the peaks found in the sample analysis (area of RSV + area of all the degradants formed in the degradation) and found to be in between 98.1 and $99.3 \%$ (a)

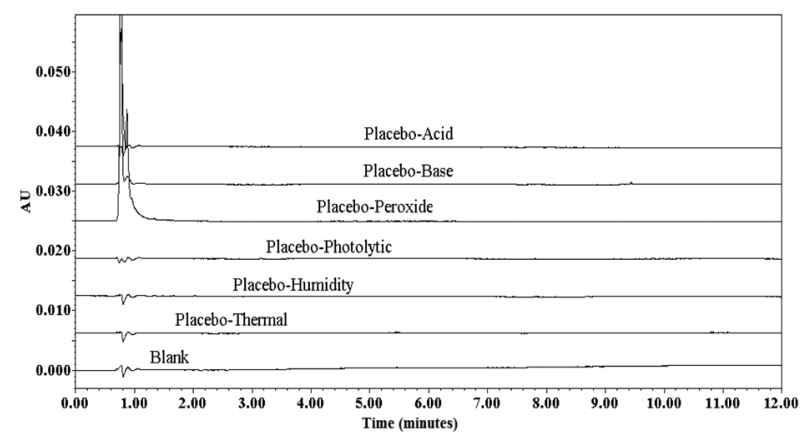

(b)

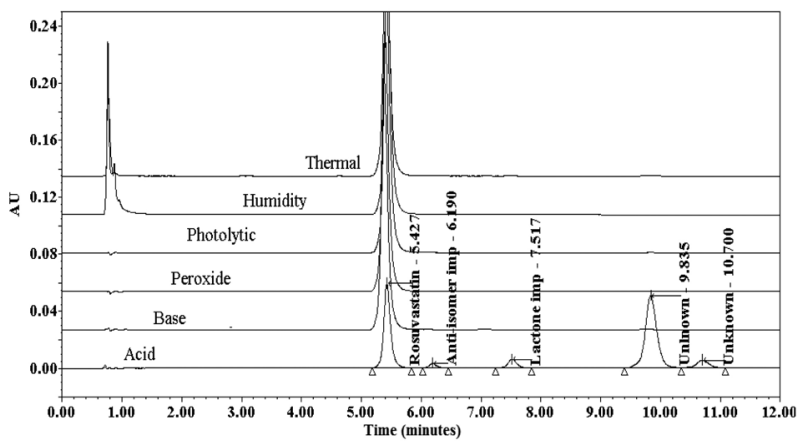

Figure 3. Typical chromatograms of RSV degradation placebo samples (a) and forced degradation samples $(b)$

(Table 2). The assay of RSV was unaffected in presence of impurities and degradation products thus confirm the stability-indicating of the developed UPLC method.

\section{Analytical parameters and validation}

After satisfactory development of method RSV tablet powder was subjected to method validation according to ICH guidelines. ${ }^{17}$ The method was validated to demonstrate its suitability for intended purpose using the standard procedure and the validation characteristics including specificity, accuracy, precision, robustness, LOD, LOQ, linearity and stability have been evaluated.

Table 2. Forced degradation results of RSV tablets

\begin{tabular}{|c|c|c|c|c|c|}
\hline Degradation conditions & $\%$ Assay for RSV & $\%$ Anti-isomer impurity & $\%$ Lactone impurity & $\%$ Major degradation product & Mass balance \\
\hline $\begin{array}{l}\text { Acid treatment } \\
(1 \mathrm{~N} \mathrm{HCl}, 3 \mathrm{~h} \text { at room temperature) }\end{array}$ & 62.7 & 0.59 & 7.12 & 30.02 & 98.6 \\
\hline Base treatment $\left(1 \mathrm{~N} \mathrm{NaOH}, 70^{\circ} \mathrm{C}, 2 \mathrm{~h}\right)$ & 98.3 & 0.02 & 0.03 & 0.11 & 98.1 \\
\hline $\mathrm{H}_{2} \mathrm{O}_{2}$ treatment $\left(5 \% \mathrm{H}_{2} \mathrm{O}_{2}, 70{ }^{\circ} \mathrm{C}, 2 \mathrm{~h}\right)$ & 97.5 & 0.02 & 0.05 & 0.34 & 98.9 \\
\hline Thermal- $105^{\circ} \mathrm{C}, 7$ days & 98.7 & 0.02 & 0.04 & 0.55 & 98.3 \\
\hline Humidity-90\% RH, $25^{\circ} \mathrm{C}, 7$ days & 99.1 & 0.03 & 0.03 & 0.17 & 99.0 \\
\hline Photolytic-1.2 m lux h, $200 \mathrm{Wh} / \mathrm{m}^{2}, 7$ days & 99.5 & 0.01 & 0.03 & 0.15 & 99.3 \\
\hline
\end{tabular}




\section{System suitability}

The main purpose to perform system suitability was to check the resolution between RSV and anti-isomer and was found to be greater than 2.5. The percentage RSD for an average area of six replicate injections of diluted standard was found to be below 0.24 , the tailing factor was less than 1 and the theoretical plates were around 13000 .

\section{LOD and LOQ}

The concentration with signal to noise ratio of at least 3 was taken as LOD and concentration with signal to noise ratio of at least 10 was taken as LOQ, which meets the criteria defined by ICH guidelines. ${ }^{17}$ The LOD and LOQ results of RSV and known impurities were presented in Table 3.

Table 3. LOD, LOQ, linearity and precision data

\begin{tabular}{lccc}
\hline Parameter & RSV & $\begin{array}{c}\text { Anti-isomer } \\
\text { impurity }\end{array}$ & $\begin{array}{c}\text { Lactone } \\
\text { impurity }\end{array}$ \\
\hline LOD $\left(\mu \mathrm{g} \mathrm{mL}^{-1}\right)$ & 0.005 & 0.007 & 0.008 \\
S/N ratio & 2.7 & 3.1 & 3.2 \\
LOQ $\left(\mu \mathrm{g} \mathrm{mL}^{-1}\right)$ & 0.023 & 0.020 & 0.025 \\
S/N ratio & 9.8 & 10.2 & 10.6 \\
Regression statistics & & & \\
Slope (b) & 155378 & 187029 & 184535 \\
Intercept (a) & 2736.2 & -3722.5 & -1020.2 \\
Correlation coefficient & 0.9996 & 0.9995 & 0.9998 \\
Method precision $(\% \mathrm{RSD})^{\mathrm{a}}$ & 1.12 & 1.35 & 1.54 \\
Intermediate precision $(\% \mathrm{RSD})^{\mathrm{a}}$ & 0.96 & 0.78 & 0.93 \\
\hline
\end{tabular}

Linearity range was LOQ-150\% with respect to $500 \mu \mathrm{g} \mathrm{mL}^{-1}$ of RSV for related impurities; linearity range was $50-150 \%$ with respect to $50 \mu \mathrm{gLL}^{-1}$ of RSV for assay. ${ }^{a}$ calculated by 6 determinations.

\section{Linearity}

To demonstrate the linearity of detector response for RSV, antiisomer and lactone, injected the solutions of concentrations ranging from LOQ level to $150 \%$ of the target concentration of RSV and its impurities. Plotted a graph between peak area and concentration, and results are summarized in Table 3 .

For the assay method, the linearity study was executed by injecting RSV at 5 different concentration levels i.e. 50, 75, 100, 125 and $150 \%$ to the target sample concentration. Calculated the correlation co-efficient and found to be greater than 0.999 indicates that magnificent correlation between the analyte concentration and the peak area.

\section{Precision}

System precision

The results of system precision were presented in Table 3 . The percentage RSD of area count of 6 replicate injections was below $1.6 \%$. Low values of RSD indicate that the method was precise.

\section{Method precision}

The percentage RSD for the area of anti-isomer and lactone impurities, and assay results obtained from the intermediate precision study were within $1.0 \%$, confirms good precision of the method. The calculated percentage RSD values were presented in Table 3.

\section{Accuracy}

The recovery results for assay as well as impurities were expressed in terms of mean percentage RSD. The percentage recoveries obtained for RSV, anti-isomer and lactone were within $1.9 \%$ and the results were presented in Table 4 . The recovery results indicate that the method is accurate and also found that there was no interference due to the presence of excipients in the tablet formulations.

Table 4. Accuracy results

\begin{tabular}{|c|c|c|c|c|}
\hline \multirow[t]{2}{*}{ Amount added } & \multicolumn{3}{|c|}{$\%$ Recovery range for triplicate injections } & \multirow[t]{2}{*}{$\% \mathrm{RSD}^{\mathrm{c}}$} \\
\hline & $\mathrm{RSV}^{\mathrm{a}}$ & $\begin{array}{c}\text { Anti-isomer } \\
\text { impurity }^{\mathrm{b}}\end{array}$ & $\begin{array}{l}\text { Lactone } \\
\text { impurity }^{\mathrm{b}}\end{array}$ & \\
\hline LOQ & $99.1-100.3$ & $98.3-100.2$ & $97.8-99.4$ & 0.78 \\
\hline $50 \%$ & $99.3-99.8$ & $97.3-99.7$ & $98.2-99.5$ & 1.54 \\
\hline $100 \%$ & $99.7-100.8$ & $98.6-99.4$ & $98.7-99.4$ & 1.26 \\
\hline $150 \%$ & $99.6-100.5$ & $99.1-99.6$ & $99.2-99.8$ & 1.88 \\
\hline
\end{tabular}

a $\%$ Recovery obtained from assay; ${ }^{\mathrm{b}} \%$ Recovery obtained from related substances; ${ }^{\mathrm{c}}$ Mean of triplicate determination at each level.

\section{Robustness}

In all robust conditions, the resolution between RSV and antiisomer has been found to be greater than 2.0 and the resolution between anti-isomer and lactone impurity was not less than 5.0. The peak shapes are found to be good for all impurities and RSV in all conditions, the assay variability was within $\pm 2.0 \%$ and the discrepancy from the estimation of RSV impurities was within $\pm 7.0 \%$. So, the method was found to be robust with respect to variability in all robust conditions.

\section{Stability of sample solution}

The results of solution stability of RSV tablets indicate that lactone and anti-isomer impurities were found to be stable up to 12 and $24 \mathrm{~h}$, respectively. The sample solutions have used for analysis within $12 \mathrm{~h}$ after preparation.

\section{Identification of the unknown major degradant from acid degradation}

To identify an unknown major degradant formed in acid degradation sample UPLC-MS analysis was performed by coupling UPLC with QToF mass spectrometer using the same mobile phase. In UPLC-MS analysis, RSV shows the molecular ion at $\mathrm{m} / z, 482$ and the unknown degradant at $\mathrm{m} / z, 496$ which was 14 mass units higher than RSV and matched with the methylene group $\left(-\mathrm{CH}_{2}\right)$. MS/MS study was performed for both RSV and unknown degradation impurity using product ion mode by applying collision energy of about $20 \mathrm{eV}$. The MS/MS spectrum of the unknown degradant (Figure 4) shows the molecular ion at $\mathrm{m} / \mathrm{z} 496.6$ and the major fragments were detected at $m / z$ 478.2, 460.3, 270.4 and 258.5. The fragments at $\mathrm{m} / \mathrm{z} 478.2$ and 460.3 were due to loss of one and two water molecules, respectively, from the molecular ion. The fragment at $\mathrm{m} / \mathrm{z} 270.4$ was due to loss of $-\mathrm{SO}_{2} \mathrm{CH}_{3}$ along with side chain $-\mathrm{C}_{6} \mathrm{H}_{11} \mathrm{O}_{4}$ and this fragment loses $-\mathrm{CH}_{3}$ group to from a peak at $\mathrm{m} / \mathrm{z}$ 258.5. The fragmentation pattern of the unknown degradant clearly indicates that it was formed due to the esterification of the acid group present in the RSV.

\section{CONCLUSIONS}

A novel isocratic RP-UPLC method was developed for the quantification of RSV as well as its related impurities in pharmaceutical 


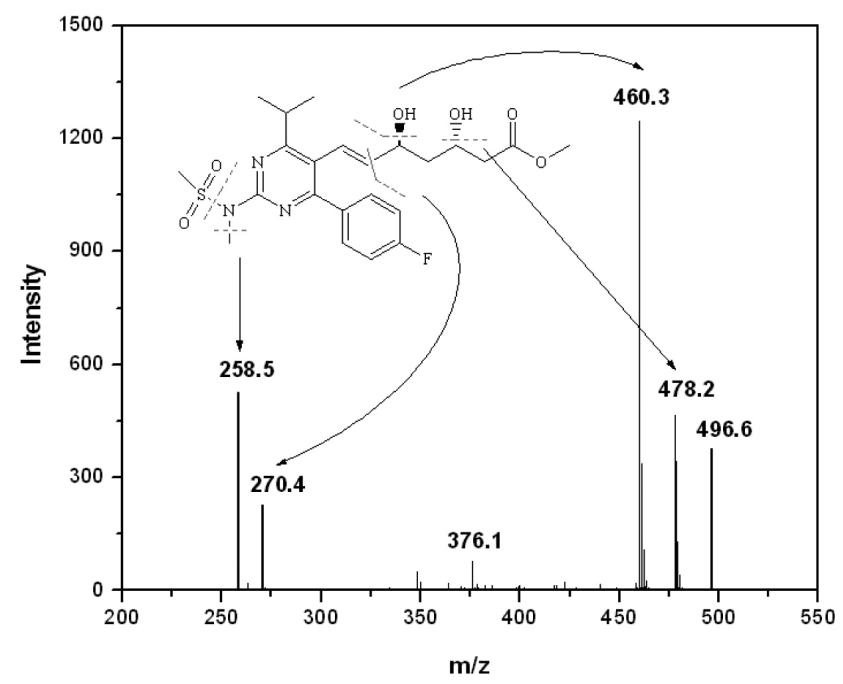

Figure 4. MS/MS spectra of degradation impurity

dosage forms in routine analysis. Degradation behaviour of the RSV was studied under various degradation stress conditions. One major degradant found in acid stress condition was identified as RSV methyl ester. The mobile phase was mass compatible and this method can also be applicable in LC-MS. All the process related impurities and the degradation impurities were well separated from the RSV revealed the stability indicating capability of the method.

\section{ACKNOWLEDGEMENTS}

The authors wish to thank to Jubilant organosys (Noida, UP, India) for their support to provide the samples and encouragement.

\section{REFERENCES}

1. Lennernas, H.; Fager, G.; Clin. Pharmacokinet 1997, 32, 403.

2. McTaggart, F.; Bucket, L.; Davidson, R.; Holdgate, A.; McCormick, A.; Schneck, D.; Smith, G.; Warwick, M.; Am. J. Cardiol. 2001, 87, 28 B.

3. Kivisto, K. T.; Kantola, T.; Neuvonen, P. J.; Br. J. Clin. Pharmacol. 1998, 46, 49.

4. Neuvonen, P. J.; Kantola, T.; Kivisto, K. T.; Clin. Pharmacol. Ther. 1998, 63, 332

5. Sivakumaran, M. S.; Ramesh, D.; Sarma, M. S. P.; Sukumar, N.; Gangadhar, B. S. N.; Narayan, K. A. S. S.; Sunil Kumar, B.; US pat. 201000488992010 (AC07D23942F).

6. Jerkovich, A. D.; LoBrutto, R.; Vivilecchia, R. V.; LC-GC North. Am. Suppl. 2005, 23, 15.

7. Leandro, C. C.; Hancock, P.; Fussel, R. J.; Keely, B. J.; J. Chromatogr., A 2006, 1103, 94.

8. Jin, H.; Praveen Kumar, A.; Paik, D. H.; Ha, K. C.; Yoo, Y. J.; Lee, Y. I.; Microchem. J. 2010, 94, 139.

9. Gross, M. L.; J. Am. Soc. Mass Spectrom. 1994, 5, 57.

10. Lacorte, S.; Fernandez-Alba, A. R.; Mass Spectrom. Rev. 2006, 25, 866.

11. Suslu, I.; Celebier, M.; Altinoz, S.; Chromatographia 2007, 766, S65.

12. Uyar, B.; Celebier, M.; Altinoz, S.; Pharmazie 2007, 62, 411.

13. Gupta, A.; Mishra, P.; Shah, K.; E-J. Chem. 2009, 6, 89.

14. Hull, C. K.; Penman, A. D.; Smith, C. K.; Martin, P. D.; J. Chromatogr., B: Anal. Technol. Biomed. Life Sci. 2002, 772, 219.

15. Lan, K.; Jiang, X. H.; Li, Y. X.; Wang, L.; Zhou, J.; Jiang, Q.; Ye, L.; J. Pharm. Biomed. Anal. 2007, 44, 540.

16. Mehta, T. N.; Patel, A. K.; Kulkarni, G.; Subbaiah, G.; J. AOAC Int. 2005, $88,1142$.

17. ICH - Harmonised Tripartity Guideline; Validation of Analytical Procedures: Text and Methodology Q2(R1), IFPMA: Geneva, 2005

18. Bakshi, M.; Singh, S.; J. Pharm. Biomed. Anal. 2002, 28, 1011. 